\title{
Description of a "Trans-Saharan" Strain of Human T-Lymphotropic Virus Type 1 in West Africa
}

\author{
Gianguglielmo Zehender, PhD, BS, * Erika Ebranati, BS, * Chiara De Maddalena, BS, * \\ Erika Gianelli, MD, * Agostino Riva, MD, * Stefano Rusconi, MD, * Benedetta Massetto, MD, * \\ Fanny Rankin, MD, $†$ Manuel Acurie, MD, $\dagger$ and Massimo Galli, MD*
}

Summary: The aim of this study was to assess the prevalence and the molecular epidemiology of human T-lymphotropic virus type 1 (HTLV-1) in a group of pregnant women living in Guinea Bissau. We studied 427 consecutive pregnant women attending 10 centers for HIV-1 infection monitoring in Bissau. HTLV-1 infection was found in $2.6 \%$ of the patients. Phylogenetic analysis of the long terminal repeat region showed that 10 isolates were of the cosmopolitan subtype (HTLV-1a) and that only 1 was of the widespread Central African subtype (HTLV-1b). All the cosmopolitan isolates belonged to the HTLV-1aD subgroup, which was first described in North Africa and clustered with other Senegal and Guinea isolates to form a significant West African clade. Our data show a high prevalence of HTLV-1 in Guinea Bissau and suggest the existence of a trans-Saharan strain distributed in North and West Africa, which probably crossed the desert in the past as a result of contacts between nomadic and sedentary populations or along trading routes.

Key Words: Guinea Bissau, human T-lymphotropic virus type 1, molecular epidemiology, northern and western Africa, phylogenetic analysis

(J Acquir Immune Defic Syndr 2008;47:269-273)

U nlike other retroviruses, human T-lymphotropic virus type 1 (HTLV-1) is genetically conserved and has a slow evolutionary rate that has recently been estimated as being between $10^{-7}$ and $10^{-6}$ substitutions per site per year for env and long terminal repeat (LTR). ${ }^{1}$ Phylogenetic analyses of viral genes have shown the existence of 6 HTLV-1 subtypes (a to f) characterized by a mean sequence divergence of $<10 \%$ and a different geographic distribution. Five of these can be detected in African populations ${ }^{2}$ : subtype $1 \mathrm{~b}$ is the most widespread subtype in Central Africa; subtypes 1d, 1e, and 1f

Received for publication November 15, 2007; accepted December 24, 2007. From the *Department of Clinical Sciences "L. Sacco," Section of Infectious Diseases, University of Milan, Milan, Italy; and the †Associação Céu e Terras, Bissau, Guinea Bissau.

Partially supported with grant 45G.14 from the Italian Concerted Action for the development of a Vaccine against HIV/AIDS

Correspondence to: Gianguglielmo Zehender, PhD, BS, Dipartimento di Scienze Cliniche "L.Sacco," Sezione Malattie Infettive, Università di Milano, c/o Ospedale L. Sacco, Via G.B. Grassi 74, 20157 Milano, Italy (e-mail: gianguglielmo.zehender@unimi.it).

Copyright (c) 2008 by Lippincott Williams \& Wilkins have been isolated in pygmies living in the same region; ${ }^{3-5}$ and the 1a (cosmopolitan) subtype is found all over the world.

Analysis of the LTR of subtype 1a allows the recognition of 5 divergent subgroups: HTLV-1aA (transcontinental) is found at different prevalence rates throughout the world and has been identified in South Africa, $1 \mathrm{aB}$ is reported in Japan and in subjects of Japanese descent in the Americas, $1 \mathrm{aC}$ has been reported in Côte d'Ivoire and Ghana as well as in people living in Central and South America, $1 \mathrm{aD}$ was first isolated in North Africa, ${ }^{4,6}$ and $1 \mathrm{aE}$ has been reported in subjects of African descent living in Peru. ${ }^{7}$

HTLV-1 is widespread in sub-Saharan Africa, particularly in West and Central Africa. In West Africa, the reported rates of seropositivity for anti-HTLV-1 antibodies vary from $1 \%$ to more than $5 \%$ among blood donors and/or pregnant women in Ghana, ${ }^{8}$ Benin,,${ }^{9}$ Burkina Faso, ${ }^{10}$ Mali, ${ }^{11}$ Senegal, ${ }^{12}$ Nigeria, ${ }^{13}$ and Guinea Bissau. ${ }^{14}$

The prevalence of HTLV-1 infection in Guinea Bissau is one of the highest in Africa. A serosurvey carried out in the year 2000 found that $9.3 \%$ of blood donors and $3.6 \%$ of the general population of Bissau were antibody-positive, ${ }^{15}$ whereas the prevalence in pregnant women was $3.3 \%$ in $1992 .{ }^{16}$ Little is still known about the distribution of HTLV-1 subtypes/subgroups in the country.

The aim of this study was to assess the prevalence of HTLV-1 infection in a consecutive series of pregnant women attending a counseling and assistance program for the prevention of the vertical transmission of HIV infection and to investigate its molecular epidemiology in this highly infected area.

\section{MATERIAL AND METHODS}

\section{Patients}

A total of 427 blood samples were obtained from a consecutive series of pregnant women of different ethnic groups from Bissau and the surrounding region who were attending 10 centers for the prevention of mother-to-child transmission of HIV in the town of Bissau. Their mean age was 23.2 years $(\mathrm{SD}=5.2)$. All the main ethnic groups of Bissau were represented in our case file (19\% Fula, 13\% Mandinga, $12 \%$ Papel, $18.8 \%$ Balanta, and $<10 \%$ others). Guidelines of the Guinea-Bissau Ministry of Health were observed, and informed consent forms were obtained. 


\section{Methods}

Approximately $100 \mu \mathrm{L}$ of whole blood was spotted onto filter papers (Guthrie cards), which were allowed to dry overnight at room temperature before being frozen at $-30^{\circ} \mathrm{C}$ until use.

Eluates from the filter papers were screened for HTLV antibodies using an enzyme-linked immunosorbent assay (ELISA; Murex HTLV I+II; Murex Biotech Limited, Dartford, United Kingdom). Whole genomic DNA was purified from the dried blood using a commercial kit (Generation Capture Card Kit; Gentra Systems, Minneapolis, MN) and tested for HTLV DNA by amplifying LTR and tax regions using different polymerase chain reaction (PCR) protocols.

The nested PCR protocols used for LTR (nt 4 to 697) and $\operatorname{tax}$ (nt 7358 to 7516) amplification have been described elsewhere. ${ }^{17}$

The LTR PCR fragments were purified using spin columns (QIAquick PCR Purification Kit; Qiagen, Hilden, Germany) and directly sequenced in both directions in an automated sequencing apparatus (ABI Prism 3100 Genetic Analyser; Applied Biosystems, Foster City, CA) using forward and reverse inner primers and the dideoxy chain termination method (BigDye Terminator Cycle Sequencing Reaction Mixture; Perkin-Elmer, Foster City, CA).

The sequences were submitted to the GenBank with assigned accession numbers (EU401868-EU401878).

\section{Phylogenetic Analysis}

Phylogenetic analyses were made of LTR sequences (630 nucleotides in length). Three LTR sequences (GB2722, GB3484, and GB2304) were shorter than the others ( 375 base pairs [bp] for GB2722 and 608 bp for GB3484 and GB2304) and were analyzed separately.

The patients' isolates were aligned with reference sequences retrieved from the Genbank using CLUSTAL W. ${ }^{18}$

The best-fitting substitution models and parameters were estimated using a hierarchical likelihood ratio test implemented using the MODELTEST 3.06 computer program. ${ }^{19}$ The selected model was that of Tamura and $\mathrm{Nei}^{20}$ with discrete $\gamma$-distributed rates among sites $(\alpha$ shape $=0.5)$. Phylogeny was reconstructed using the neighbor joining (NJ) clustering method and maximum likelihood (ML) analysis. Only the topologies confirmed by the 2 methods were considered significant. The phylogenetic reconstruction, trees, and genetic distance statistics were obtained using MEGA version 3.1 ${ }^{21}$ and PAUP* version 4.0b10 (D. Swofford, Sinauer Associates, Inc., Sunderland, MA).

The significance of the interior branches in the NJ tree was tested by means of bootstrap analysis with 1000 replicates; bootstrap values of $\geq 60 \%$ were considered significant. Branch length significance was estimated using the likelihood ratio test for a branch length of 0 , implemented in PAUP* ( $P \leq 0.05$ significance).

\section{Strains Used for the Phylogenetic Analyses}

The names and accession numbers of the reference strains used for LTR phylogenetic analysis were as follows: CH26 (D23690), GB233 (D23692), AINU(D23694), pGH78 (D23693), ATK1 (J02029), RKI4 (AF054627), RK15
(AY854249), Qu1 (Y16475), Qu2 (Y16476), Qu3 (Y16477), Me1 (Y16478), Me2 (Y16479), Me3 (Y16480), Nar (AF063820), B11 (Y16481), B12 (Y16482), B13 (Y16483), Ni1 (Y16484), Ni2 (Y16487), HS35 (D13784), MT2 (L03562), Boi (L36905), TBH2 (L76025), TBH5 (L76027), H23 (L76312), StDen (L76306), GAB7 (L76311), PH236 (L76307), T49 (L76305), 12503 (L76309), H24 (L76308), ELzaire (S74562), mel5 (L02534), BO (U12804), OD (U12805), Pr52 (U12806), Pr144 (U12807), JCP (X88875), efe1 (Y17014), Lib2 (Y17017), MOMJ (Z31659), MT4 (Z31661), MWMG (Z31662), ITIS (Z32527), FrGu1 (AY324785), PH906 (AY342303), PH 907 (AY342304), 6Aissa (AY342300), 2BAbdou (AY342302), 5BMarieto (AY342301), PH908 (AY342305), PH979 (AY342306), BD89517 (DQ235699), and NM1626 (AF063821).

\section{Statistical Analysis}

Significances were tested using the Student $t$ test for comparisons of mean values and the Pearson $\chi^{2}$ test for comparisons of proportions. All the analyses were made using the SPSS package (SPSS version 11; SPSS Inc., Chicago, IL).

\section{RESULTS}

Eleven (2.6\%) of the 427 samples analyzed were reactive to the anti-HTLV-1/2 ELISA, and all of them had positive results to tax and LTR PCR amplification. The characteristics of the 11 HTLV-1-positive subjects are shown in Table 1.

Analysis of the LTR sequences in the 11 isolates with 54 reference sequences representing all the known HTLV-1 subtypes and subgroups showed 6 significant clades corresponding to the known HTLV-1 subtypes (HTLV-1a, HTLV-1b, HTLV-1c, HTLV-1d, HTLV-1e, and HTLV-1f). Ten patient isolates (including the shorter GB2722, GB3484, and GB2304) belonged to the cosmopolitan subtype (HTLV-1a); the other isolate (obtained from a Fula patient) was included within the Central African subtype HTLV-1b (Fig. 1). The cosmopolitan subtype segregated into 5 significant monophyletic groups corresponding to the known subgroups A to E:

TABLE 1. Demographic and Virologic Characteristics of the Women From Whom the 11 Typed HTLV-1 Isolates Were Obtained

\begin{tabular}{cclc}
\hline Isolate & Age $(\mathbf{y})$ & Ethnic Group & HIV-1 \\
\hline GB2304 & 18 & Mandinga & $\mathrm{N}$ \\
GB2383 & 18 & Papel & $\mathrm{N}$ \\
GB2412 & Fula & $\mathrm{N}$ \\
GB2524 & 29 & Mancanha & $\mathrm{N}$ \\
GB2604 & 30 & Mandinga & $\mathrm{P}$ \\
GB2722 & 21 & Mansonca & $\mathrm{N}$ \\
GB3474 & 32 & Fula & $\mathrm{N}$ \\
GB3484 & 26 & Fula & $\mathrm{N}$ \\
GB4772 & 35 & Balanta & $\mathrm{N}$ \\
GB10533 & 20 & Mixed & $\mathrm{P}$ \\
GB12313 & 16 & Felupe & $\mathrm{N}$ \\
\hline
\end{tabular}

$\mathrm{N}$ indicates negative; $\mathrm{P}$, positive 


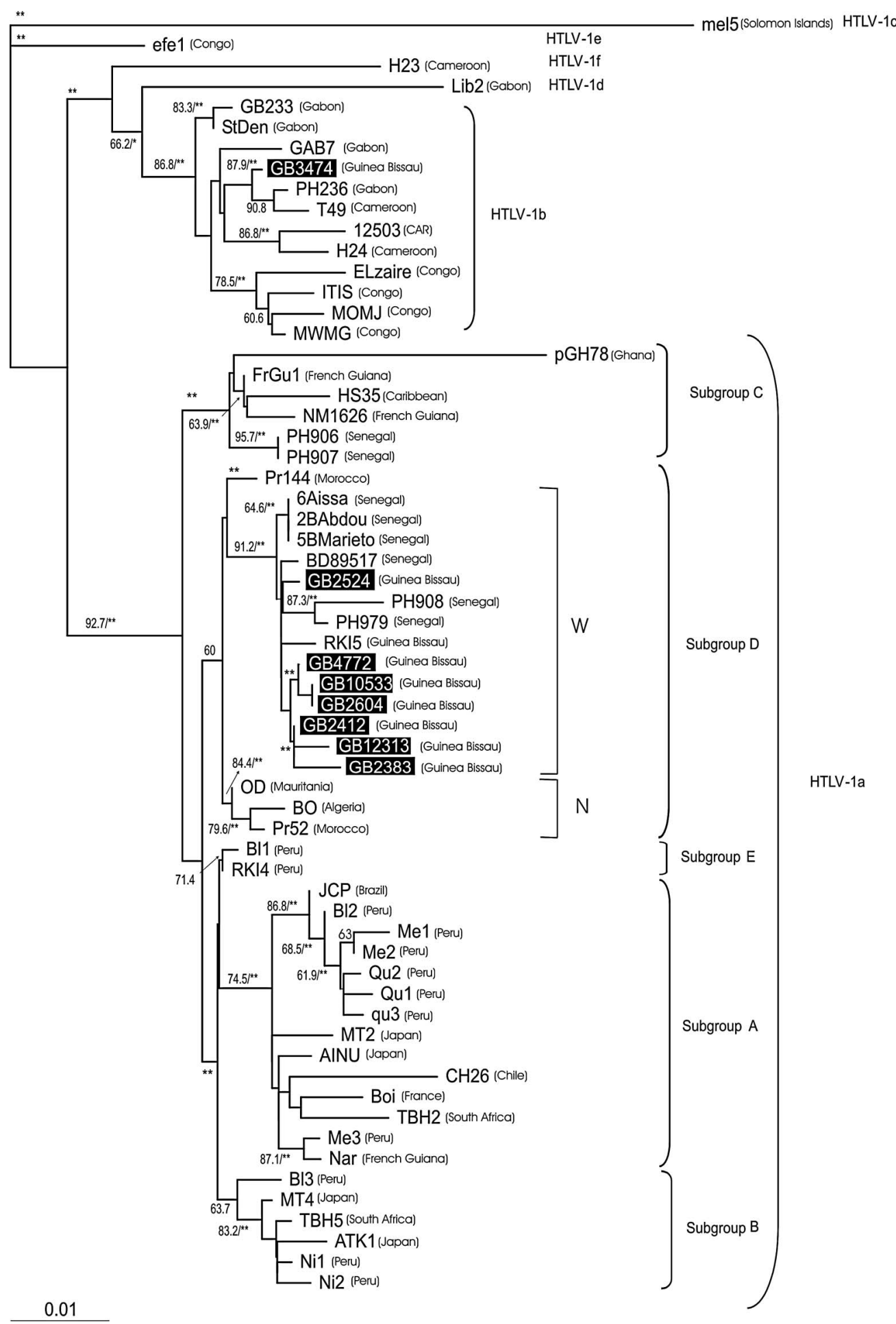

FIGURE 1. NJ tree of a 630-bp fragment of the HTLV-1 LTR region. The tree was rooted using Melanesian strain HTLV-1c (Mel5) as the outgroup, and the numbers on the branches give the bootstrap percentage values using 1000 replicates (bootstrap values $\geq 60 \%)$; the asterisks indicate that the branch length was statistically significant on $\mathrm{ML}$ testing $\left({ }^{\star} 0.001<P \leq 0.05 ;{ }^{* \star} P \leq\right.$ $0.001)$. Lower case characters indicate the strains grouping in different subtypes, and upper case characters indicate the strains grouping in different HTLV-1a subgroups. The 2 clades of HTLV-1aD have been highlighted. N indicates North African clade; W, West African clade. The isolates described in the text are shaded. Shorter sequences (GB2722, GB3484, and GB2304) were not included in this analysis. 
all 10 cosmopolitan isolates were in subgroup $1 \mathrm{aD}$ (corresponding to the North African clade). In particular, they formed a significant cluster with another 7 isolates from Guinea Bissau and Senegal retrieved from databases. The HTLV-1aD subgroup included 2 significant clades: one encompassing most isolates from Mauritania, Morocco, and Algeria, supported by an NJ bootstrap value of $84.4 \%(P \leq$ 0.001 at ML analysis) and the other including strains from Senegal and Guinea Bissau, with a bootstrap value of $91.2 \%$ $(P \leq 0.001$; see Fig. 1). One strain from Morocco (Pr144) formed an outgroup of the Senegal/Guinean clade. The mean genetic distance between the 2 clades (North and West African) within HTLV- $1 \mathrm{aD}$ was $1.2 \%$ compared with a mean of $2.0 \%$ from HTLV- $1 \mathrm{aB}$ and $2.5 \%$ from HTLV- $1 \mathrm{aC}$.

Analysis of the nucleotides of the HTLV-1aD LTR sequences showed that the isolates from Senegal and Guinea Bissau differed from the North African strains in terms of 3 mutations at nucleotides C367A, A547C, and T615C. Analysis of the 3 shorter sequences showed that they were all included in the Senegal/Guinean clade and confirmed the presence of the 3 mutations.

\section{Supplementary Epidemiologic Data}

HIV-1 infection was significantly more frequent among the HTLV-1-positive women than among the HTLV-1negative women $(2[18.2 \%]$ of 11 women vs. 20 [4.8\%] of 416 women; $P=0.04$ by $\chi^{2}$ test).

There was no significant difference in the distribution of the ethnic groups among the HTLV-1-positive or -negative subjects, although an excessive number of Fula and Mandinga subjects were observed among the former (see Table 1).

\section{DISCUSSION}

Africa represents the cradle of primate T-lymphotropic viruses $^{2}$ and hosts most HTLV-1 subtypes and subgroups. Our data show that the cosmopolitan subtype of HTLV-1 (HTLV1a) is prevalent in Guinea Bissau, because only 1 of our 11 isolates was not cosmopolitan but belonged to the most prevalent subtype in Central Africa (HTLV-1b).

Guinea Bissau occupies an area of approximately $36,000 \mathrm{~km}^{2}$ and has a population of 1.6 million people characterized by a high degree of cultural and genetic heterogeneity. There are a number of ethnic groups with different languages, religions, and cultures, many of which are also living in bordering countries. In particular, the Fula are a formerly seminomadic population of approximately 30 million individuals now dispersed throughout the Sahel region (Nigeria, Guinea Conakry, Chad, and Cameroon). ${ }^{22}$ Interestingly, this was the most frequently represented ethnic group among the HTLV-1-infected subjects, and the only isolate belonging to the Central African subtype came from a Fula subject.

The presence of HTLV-1 has been reported in various Sahara countries, particularly Morocco, ${ }^{23}$ Tunisia, ${ }^{24}$ Egypt, ${ }^{25}$ Mali, ${ }^{11}$ Mauritania, and Algeria, and LTR analyses of some of these isolates have identified the North African subgroup of the cosmopolitan subtype (HTLV-1aD). ${ }^{6}$
The phylogenetic analyses of all the cosmopolitan isolates from our patients, together with other previously described isolates from Guinea Bissau and Senegal, ${ }^{12,26}$ showed that they belonged to the HTLV-1aD subgroup. In comparison with the North African isolates, the sub-Saharan sequences clustered together, and 2 significant clades (one including all but 1 North African isolate and the other including all the Senegal/Guinean isolates) were observed in the HTLV-1aD subgroup. The mean divergence between northern and western isolates was $1.2 \%$ in LTR, which is less than the reported divergence among HTLV-1 subtypes and based on 3 polymorphic sites. Given the low evolutionary rate of the virus, ${ }^{1}$ it can be postulated that the segregation of the northern and western clades took a long time to produce such a degree of divergence.

Taken together, these observations support the existence of a trans-Saharan HTLV-1 strain that includes 2 clades: one from North Africa and the other from West Africa. Significant contacts between Berber tribes and West African sub-Saharan populations have been documented since ancient times. ${ }^{27}$ The mixture of cultures increased around the 11th century, when the southern populations were progressively converted to Islam and empires, such as the Ghana and Mali empires at the southern terminus of the caravan routes, successively expanded and disappeared. ${ }^{22}$ The trans-Saharan routes were extensively used for the slave trade from south to north, ${ }^{28}$ and sea routes also contributed to the slave trade to Morocco and Mediterranean North Africa. These multiple complex relations may explain the existence of a trans-Saharan HTLV-1 subgroup, although its geographic origin remains an enigma. The existence of a western clade in the HTLV-1aD subgroup that was separate from a northern clade may have been attributable to different phenomena. It is possible to hypothesize the past arrival in West Africa of a strain of HTLV-1aD coming from North Africa. Nevertheless, the high prevalence of HTLV-1aD in Guinea Bissau seems to support the hypothesis that this area was the source of the variant, because this subgroup is clearly the main contributor to the country's disproportionate prevalence of HTLV-1 infection. For this reason, a second hypothesis may be that the western clade was attributable to the rapid expansion of 1 viral strain as the result of a recent increase in population risk and/or changes in the main route of transmission. Larsen et $\mathrm{al}^{15}$ found a 3.6\% prevalence of HTLV-1 infection among adults living in Guinea Bissau, particularly among HIV-positive women and, in agreement with other authors, ${ }^{14}$ concluded that sexual transmission played a central role in spreading the infection in the country, probably amplified by various parenteral risk factors as has been postulated in the case of other retroviruses such as HIV-2. ${ }^{29}$ Our data show a prevalence of $2.6 \%$ among pregnant women and a frequency of HIV-1 infection significantly higher among those who were HTLV-1-positive.

In conclusion, our data, confirming the high spread of HTLV-1 infection in Guinea Bissau, show that HTLV-1aD is the prevalent subgroup in this country and in Senegal, extending the area of the distribution of this strain from North to West Africa. They also suggest the existence of a transSaharan HTLV-1aD, which probably crossed the desert sometime in the past through nomadic populations or along 
trade routes. Further studies are needed to clarify the origin and the epidemiologic history of such an interesting viral strain.

\section{ACKNOWLEDGMENTS}

The authors are grateful to the Italian Organizzazione Non Lucrativa di Utilita' Sociale "Associazione Cielo e Terre" and the Guinean organization "Associação Céu e Terras" for their invaluable and continuous collaboration. The authors thank all the staff of the project "Promotion of Health and Fight Against AIDS in Guinea Bissau" and the patients who participated in the study.

\section{REFERENCES}

1. Lemey P, Pybus OG, Van Dooren S, et al. A Bayesian statistical analysis of human T-cell lymphotropic virus evolutionary rates. Infect Genet Evol. 2005;5:291-298.

2. Verdonck K, Gonzalez E, Van Dooren S, et al. Human T-lymphotropic virus 1: recent knowledge about an ancient infection. Lancet Infect Dis. 2007; 7:266-281

3. Salemi M, Van Dooren S, Audenaert E, et al. Two new human T-lymphotropic virus type I phylogenetic subtypes in seroindeterminates, a Mbuti pygmy and a Gabonese, have closest relatives among African STLV-I strains. Virology. 1998;246:277-287.

4. Yamashita M, Ido E, Miura T, et al. Molecular epidemiology of HTLV-I in the world. J Acquir Immune Defic Syndr Hum Retrovirol. 1996;13(Suppl 1):S124-S131.

5. Mahieux R, Ibrahim F, Mauclere P, et al. Molecular epidemiology of 58 new African human T-cell leukemia virus type 1 (HTLV-1) strains: identification of a new and distinct HTLV-1 molecular subtype in Central Africa and in Pygmies. J Virol. 1997;71:1317-1333.

6. Gasmi M, Farouqi B, d'Incan M, et al. Long terminal repeat sequence analysis of HTLV type I molecular variants identified in four North African patients. AIDS Res Hum Retroviruses. 1994;10:1313-1315.

7. Van Dooren S, Gotuzzo E, Salemi M, et al. Evidence for a postColumbian introduction of human T-cell lymphotropic virus type I in Latin America. J Gen Virol. 1998;79:2695-2708.

8. Armah HB, Narter-Olaga EG, Adjei AA, et al. Seroprevalence of human T-cell lymphotropic virus type I among pregnant women in Accra, Ghana. J Med Microbiol. 2006;55:765-770.

9. Dumas M, Houinato D, Verdier M, et al. Seroepidemiology of human T-cell lymphotropic virus type I/II in Benin (West Africa). AIDS Res Hum Retroviruses. 1991;7:447-451.

10. Collenberg E, Ouedraogo T, Ganame J, et al. Seroprevalence of six different viruses among pregnant women and blood donors in rural and urban Burkina Faso: a comparative analysis. J Med Virol. 2006;78:683-692.

11. Fouchard N, Mahe A, Huerre M, et al. Cutaneous T cell lymphomas: mycosis fungoides, Sezary syndrome and HTLV-I-associated adult T cell leukemia (ATL) in Mali, West Africa: a clinical, pathological and immunovirological study of 14 cases and a review of the African ATL cases. Leukemia. 1998;12:578-585.
12. Diop S, Calattini S, Abah-Dakou J, et al. Seroprevalence and molecular epidemiology of human T-cell leukemia virus type 1 (HTLV-1) and HTLV-2 in blood donors from Dakar, Senegal. J Clin Microbiol. 2006;44: $1550-1554$

13. Olaleye DO, Omotade OO, Sheng Z, et al. Human T-cell lymphotropic virus types I and II infections in mother-child pairs in Nigeria. $J$ Trop Pediatr. 1999;45:66-70.

14. Melbye M, Poulsen AG, Gallo D, et al. HTLV-1 infection in a populationbased cohort of older persons in Guinea-Bissau, West Africa: risk factors and impact on survival. Int J Cancer. 1998;76:293-298.

15. Larsen O, Andersson S, da Silva Z, et al. Prevalences of HTLV-1 infection and associated risk determinants in an urban population in Guinea-Bissau, West Africa. J Acquir Immune Defic Syndr. 2000;25:157-163.

16. Naucler A, Andersson S, Albino P, et al. Association between HTLV-1 and HIV-2 infections in Bissau, Guinea-Bissau. AIDS. 1992;6:510-511.

17. Zehender G, Colasante C, De Maddalena C, et al. High prevalence of human T-lymphotropic virus type 1 (HTLV-1) in immigrant male-tofemale transsexual sex workers with HIV-1 infection. J Med Virol. 2004 74:207-215

18. Thompson JD, Higgins DG, Gibson TJ. CLUSTAL W: improving the sensitivity of progressive multiple sequence alignment through sequence weighting, position-specific gap penalties and weight matrix choice. Nucleic Acids Res. 1994;22:4673-4680.

19. Posada D, Crandall KA. MODELTEST: testing the model of DNA substitution. Bioinformatics. 1998;14:817-818.

20. Tamura K, Nei M. Estimation of the number of nucleotide substitutions in the control region of mitochondrial DNA in humans and chimpanzees. Mol Biol Evol. 1993;10:512-526.

21. Kumar S, Tamura K, Nei M. MEGA3: integrated software for Molecular Evolutionary Genetics Analysis and sequence alignment. Brief Bioinform. 2004:5:150-163.

22. Rosa A, Brehm A, Kivisild T, et al. MtDNA profile of West Africa Guineans: towards a better understanding of the Senegambia region. Ann Hum Genet. 2004;68:340-352.

23. Farouqi B, Yahyaoui M, Alaoui FM, et al. Establishment of T-lymphoid cell lines from Moroccan patients with tropical spastic paraparesis. AIDS Res Hum Retroviruses. 1992;8:1209-1213.

24. Mojaat N, Kaabi H, Hmida S, et al. Seroprevalence of HTLV-I/II antibodies in blood donors and different groups at risk in Tunisia. J Acquir Immune Defic Syndr. 1999;22:314-315.

25. Constantine NT, Fathi Sheba M, Corwin AL, et al. A serosurvey for HTLV-I among high-risk populations and normal adults in Egypt. Epidemiol Infect. 1991;107:429-433.

26. Mahe A, Meertens L, Ly F, et al. Human T-cell leukaemia/lymphoma virus type 1-associated infective dermatitis in Africa: a report of five cases from Senegal. Br J Dermatol. 2004;150:958-965

27. Munson PJ. Archeology and the prehistoric origins of the Ghana Empire $J$ Afr Hist. 1980;21:457-466.

28. Richardson D. Review: across the desert and the sea: trans-Saharan and Atlantic slavery, 1500-1900. Hist J. 1995;38:195-204.

29. Pepin J, Plamondon M, Alves AC, et al. Parenteral transmission during excision and treatment of tuberculosis and trypanosomiasis may be responsible for the HIV-2 epidemic in Guinea-Bissau. AIDS. 2006;20 1303-1311. 